\title{
Sinciput Presentation
}

National Cancer Institute

\section{Source}

National Cancer Institute. Sinciput Presentation. NCI Thesaurus. Code C92882.

A fetal position during delivery in which the frontal part of the skull including forehead and the top of the head is first to descend into the birth canal. 